\title{
Low-Cost Air Gap Metasurface Structure for High Absorption Efficiency Energy Harvesting
}

\author{
Wei Hu, ${ }^{1,2}$ Zhao Yang, ${ }^{1}$ Fading Zhao, ${ }^{1}$ Guangjun Wen, ${ }^{1}$ Jian $\mathrm{Li}\left(\mathbb{D},{ }^{1}\right.$ Yongjun Huang $\mathbb{D},{ }^{1}$ \\ Daniele Inserra $\mathbb{B}^{1},{ }^{1}$ and Zhizhang (David) Chen $\mathbb{B}^{2,3,4}$ \\ ${ }^{1}$ School of Information and Communication Engineering, University of Electronics Science and Technology of China, \\ Chengdu 611731, China \\ ${ }^{2}$ School of Electronic Science and Engineering, University of Electronics Science and Technology of China, Chengdu 611731, China \\ ${ }^{3}$ College of Physics and Information Engineering, Fuzhou University, Fujian, China \\ ${ }^{4}$ Department of Electrical and Computer Engineering, Dalhousie University, Halifax, Nova Scotia, Canada
}

Correspondence should be addressed to Daniele Inserra; inserradaniele@uestc.edu.cn

Received 29 May 2019; Revised 20 August 2019; Accepted 12 September 2019; Published 26 September 2019

Academic Editor: Renato Cicchetti

Copyright (C) 2019 Wei Hu et al. This is an open access article distributed under the Creative Commons Attribution License, which permits unrestricted use, distribution, and reproduction in any medium, provided the original work is properly cited.

\begin{abstract}
This manuscript deals with the design of a metamaterial-based surface structure for high efficiency wireless power harvesting or collection. Differently from the previously presented structures which require the use of thicker and low-loss (and for this reason high cost) dielectric substrates, the presented work employs a dual-layer structure with a thin low-loss material and an air gap; they allow for the design of very high absorption efficiency metamaterial-based surfaces, with noticeably reduced costs. Furthermore, the air gap thickness can be used as a new degree-of-freedom (more easily adjustable than the thickness of a single-layer structure) for the optimization of other design requirements such as bandwidth or structure sizes. In comparison with other existing designs, the proposed metasurface shows a comparable absorption efficiency of $84.4 \%$ but with a larger power collection surface and lower costs.
\end{abstract}

\section{Introduction}

Wireless power transfer (WPT) concept employing radio frequency (RF) electromagnetic waves is nowadays attracting considerable interest from both academy and industry. Solar power satellite systems, high altitude platforms, electrical vehicle or unmanned aerial vehicle (UAV) remote "fueling," as well as energy delivery to inaccessible regions $[1-3]$ are only few possible applications for longrange WPT which are driving the research on this topic.

A WPT system is mainly composed of a DC power source, a DC-to-RF power conversion stage, a transmitting antenna, and an harvesting device (also called rectenna) equipped with a RF collection surface (or a receiving antenna), and one or more RF-to-DC rectifiers [2]. Since the objective of a WPT system design is the end-to-end system efficiency maximization, it is important that each WPT system stage can work properly and efficiently; i.e., (a) the
DC-to-RF power conversion is very efficient, (b) the transmitting antenna suitably shapes the radiated beam toward the collection surface, (c) the most part of the incident RF power on the collection surface is gathered and delivered to the rectifier, and (d) the rectifier can work under optimum working condition, e.g., the rectifier input power is sufficient to guarantee the maximum RF-to-DC conversion efficiency.

For what the transmitting antenna design is concerned, several beam shaping methodologies have been proposed during years for both far-field and near-field, which are usually based on the maximization of the active power flow through the collection surface $[2,4]$. In particular for nearfield WPT in the Fresnel region, different focused-beam design criteria have been discussed assuming antenna array discrete apertures as in [5], or taking into account a rigorous energy-based multiport circuit representation as discussed in [4] (to include parasitic coupling and other spurious effects). 
On the other hand, another key component of a WPT system is the power harvesting device, which has to convey an impinging RF electromagnetic wave to a rectification circuit. Single rectennas operating at microwave frequencies were initially developed [6]; however, when incorporated in an array they exhibit a reduced power conversion efficiency. Microstrip patches and spiral antenna arrays were then proposed to improve the efficiency [3].

Recently, metasurfaces have been introduced with the aim of maximizing the power conversion efficiency [7]. A metasurface-based power harvester is usually constituted by an array of metamaterial cells equipped with channeling vias, and each cell is designed to achieve outstanding performance in terms of absorption efficiency.

Many different metamaterial cell structures for energy harvesting have been reported in the literature. In [8], an electric LC cell was designed on a $2.54 \mathrm{~mm}$ thick Roger TMM10i $\left(\varepsilon_{\mathrm{R}}=9.9, \tan \delta=0.002\right)$, with measured maximum unit-cell efficiency of about 93\%. In [9], an asymmetric electric LC cell was realized on a $3 \mathrm{~mm}$ thick PTFE $\left(\varepsilon_{\mathrm{R}}=2.65\right.$, $\tan \delta=0.0007)$ with simulated maximum unit-cell efficiency of $95.3 \%$. A ground backed complementary split ring resonator (G-CSRR) was realized on a $1.524 \mathrm{~mm}$ thick Rogers RT Duroid 5880 dielectric $\left(\varepsilon_{\mathrm{R}}=2.2, \tan \delta=0.001\right)$ with a unit-cell maximum efficiency of $83 \%$ [7]. In [10], a butterflytype closed loop element was implemented on an F4B substrate $\left(\varepsilon_{\mathrm{R}}=2.65, \tan \delta=0.001\right)$ of $4 \mathrm{~mm}$ thickness with a peak efficiency of $81 \%$. Polarization insensitive structures were also developed with four rotated elements and multiple channeling vias [11-13] or opportunely placing a single channeling via $[14,15]$.

All the above cited examples are implemented on thick and low-loss dielectric substrates that increase overall costs. On the contrary, two structures developed "on air" are presented in [16] (a split ring resonator loaded with a hollow cylinder structure) and [17] (a split loop three-dimensional structure without dielectric support), respectively. These two solutions have unit-cell measured efficiencies larger than $97 \%$, demonstrating that the employment of air structures is beneficial in terms of efficiency. However, implementations presented in $[16,17]$ are complex. Furthermore, unit-cell area is only $0.026 \lambda^{2}$, which means that the collected power will be very small, making the design of a highly effective AC-to-DC rectifier very challenging. In order to alleviate this problem, an RF collection network is employed with $8 \times 8$ metasurface arrays, achieving however reduced efficiencies if compared with single unit-cell results, i.e., measured peak efficiencies of 78\% [18] and 86\% [19], respectively. Another possible strategy to increase the collected power is the design of unit cells with larger area, as it has been presented in [20] by realizing a tightly coupled antenna structure whose unit cells are about five times larger than usually employed metamaterial unit cells. Nonetheless, unit cells of this structure are designed to be directly connected to an AC-toDC rectifier, requiring very high levels of impinging power density to make the structure efficient.

Motivated by the above analysis, this paper extends our original work [21] by developing a low-cost metasurface that is based on a dual-layer structure composed of a thin and low-loss dielectric material and an air gap. The original structure in [21] already presented noticeable advantages in terms of cost with respect to other previously presented metasurface harvesters, and, in this work, it is shown to possess very good power absorption efficiency. In addition, the air gap thickness is used as a new degree-of-freedom for designing unit cell that can increase the area to collect wireless power and/or for broadening the structure bandwidth. Moreover, the employment of a common metasurface unit cell with a channeling via makes the structure suitable for being integrated with an RF combining network, further enhancing the collected power levels. An efficiency of $84.4 \%$ is experimentally achieved for an $8 \times 8$ array prototype, very close to the efficiency level previously reported in the literature, but at lower cost and with larger size for larger power collection.

\section{The Proposed Metasurface Design}

2.1. Unit Cell and Air Gap Structure. The metasurface unit cell considered in this work is called electric $L C$, and it is shown in Figure 1(a) [8] (this structure has been selected for its design simplicity and the high conversion efficiency performance [22]; nonetheless, the presented air gap structure concept can be easily extended to many other unit cells). It is constituted of one square ring with two metallic bars oriented according to the required polarization (transverse electric (TE) or transverse magnetic (TM)). As described in [22], the loop structure is capable of capturing an opportunely oriented impinging electric field, and it can be qualitatively described in terms of an equivalent circuit composed by a central capacitor (determined by the central metallic bars gap and the gap among unit cells) connected in parallel to the loop inductance. As it can be deduced by the simulated surface current in Figure 1(b), the induced current on the unit cell is then channeled by a via which passes through the dielectric layer and the air gap, and it is connected to a resistive load. The resistive load can be taken as a predetermined value [19], or it can be optimized to achieve the best efficiency [8]; in our case, it is taken as $100 \Omega$ to simplify the realization of the energy collection network.

The dielectric material is usually selected for its low-loss tangent values to enhance power efficiency. However, a thickness larger than $2.54 \mathrm{~mm}$ is usually taken for optimizing the impedance matching [8], increasing realization costs (dielectric panel costs usually increase proportionally with the thickness).

In the presented work, an air gap of height $h_{2}$ is inserted between the thin dielectric layer and the ground plane, and a dielectric material F4B $\left(\varepsilon_{\mathrm{R}}=2.2, \tan \delta=0.001\right)$ is chosen because of the low-loss property and its relatively low cost.

A comparison of a classical structure namely single-layer (with $h_{2}=0$ ) and the presented dual-layer (dielectric and air gap) is presented in Figure 2 in terms of simulated $S_{11}$ and efficiency (results have been obtained with the commercial software Ansys HFSS). In the simulation, Floquet port and periodic boundary conditions are used to simulate an infinite metasurface array, and the energy harvesting efficiency has been analyzed for a TE mode with normal 


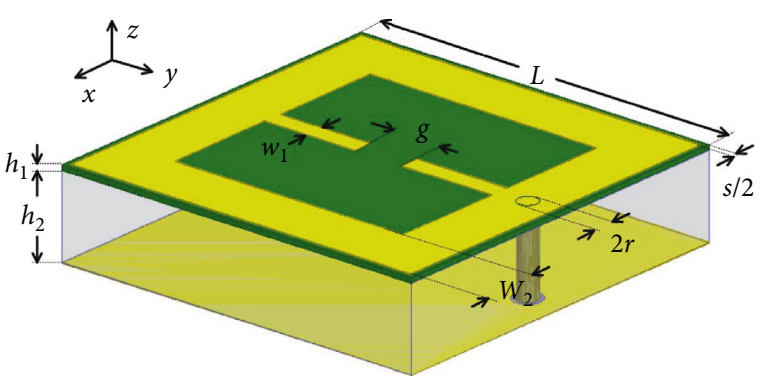

(a)

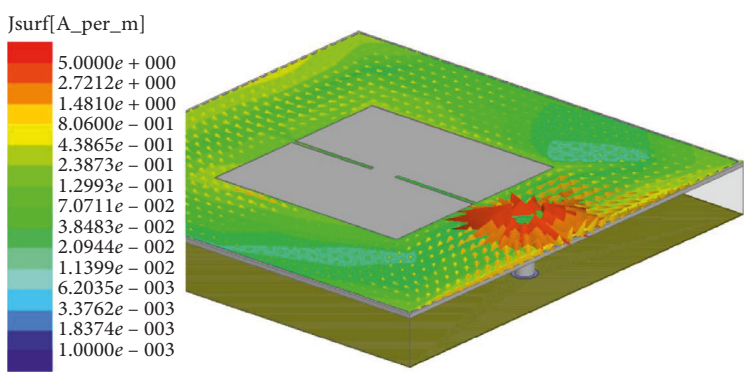

(b)

Figure 1: (a) Metasurface unit structure, and (b) simulated surface current distribution of the dual-layer structure at $2.45 \mathrm{GHz}$.

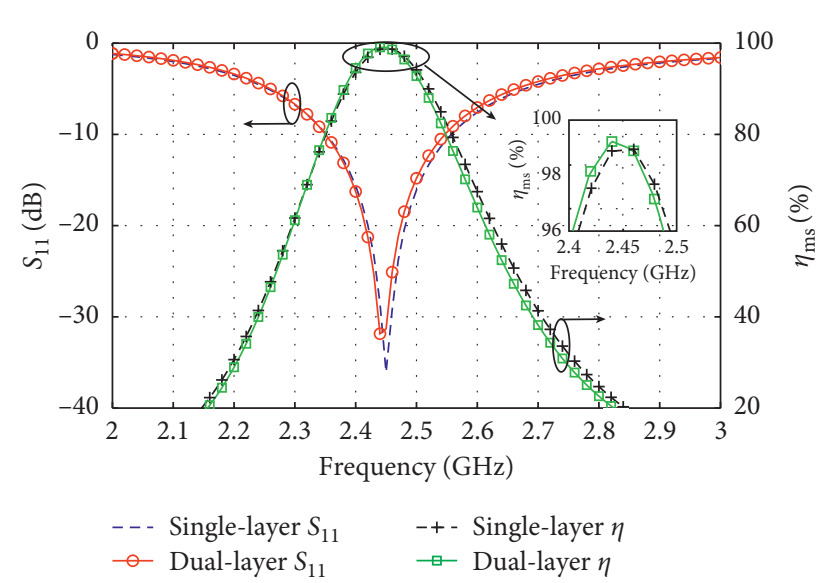

FIGURE 2: Simulated unit cell $S_{11}$ and absorption efficiency.

incidence. Furthermore, metallic parts have been taken as copper (conductivity of $58 \times 10^{6} \mathrm{~S} / \mathrm{m}^{2}$ ) with thickness $35 \mu \mathrm{m}$. Unit-cell parameters $L, s, w_{1}, w_{2}, g$, and $r$ have been optimized with the genetic algorithm optimization tool of Ansys HFSS to achieve the maximum efficiency at $2.45 \mathrm{GHz}$. The absorption efficiency is defined as

$$
\eta_{\mathrm{ms}}=\left(1-\left|\rho_{\mathrm{refl}}\right|^{2}\right) \cdot|\tau|^{2}
$$

where $\rho_{\text {refl }}$ represents the reflectivity of the metasurface to an impinging plane wave and $\tau$ is the transmission coefficient from the unit-cell surface to the resistive load [17]. Optimized unit-cell dimensions are provided in Table 1 (the via position has been fixed for simplicity as $w_{1} / 2$ ).

As it can be observed, similar performance in terms of $S_{11}$ and $\eta_{\text {ms }}$ can be achieved with the two structures (with peak efficiencies $98.9 \%$ and $99.1 \%$ for the single- and duallayer, respectively). However, it is worthy of note that the dual-layer structure can (a) be better optimized (because of an additional flexibility in the selection of the air gap thickness $h_{2}$ ), (b) better reduce the implementation costs (because of the use of a thinner dielectric substrate), (c) exploit the air gap thickness as a degree-of-freedom for the design of unit cells with broader bandwidth (as it will be shown in the next section), and (d) present a larger unit-cell area to collect more power per unit cell (in this case, it results in an increased collected power per unit cell of about $18.6 \%$ ). Finally, in Figure 3 simulated absorption efficiency performance of single- and dual-layer structures at $2.45 \mathrm{GHz}$ for
TABle 1: Optimized dimensions of the two unit cells.

\begin{tabular}{lcccccccc}
\hline Parameter & $L$ & $s$ & $w_{1}$ & $w_{2}$ & $g$ & $r$ & $h_{1}$ & $h_{2}$ \\
\hline Single-layer $(\mathrm{mm})$ & 23.6 & 0.4 & 0.5 & 6.5 & 1.8 & 0.3 & 3 & 0 \\
Dual-layer $(\mathrm{mm})$ & 25.7 & 0.3 & 0.2 & 6.3 & 1.6 & 0.5 & 0.254 & 2.746 \\
\hline
\end{tabular}

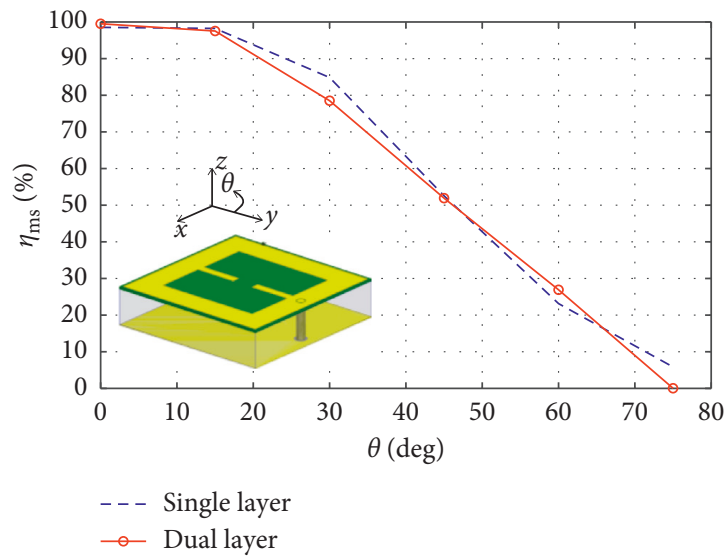

Figure 3: Simulated unit-cell absorption efficiency at $2.45 \mathrm{GHz}$ as a function of the incidence angle (TE polarization).

different incidence angles are depicted to show that the air gap does not affect the incidence angle stability.

2.2. Parametric Analysis of Air Gap Thickness. A parametric analysis of the air gap thickness $h_{2}$ has been performed (with $h_{1}=0.254 \mathrm{~mm}$ fixed), and the results of $S_{11}$ and efficiency are shown in Figure 4. The performance optimization has been carried out as described in the previous section.

As it can be observed, the increase of $h_{2}$ leads to a general bandwidth broadening. The efficiency variation is, on the other hand, unperceivable and it largely depends on the capability of the optimization tool to find a parameter combination that yields good impedance matching performance. Furthermore, it is worth noting that the unit-cell size increase is not linear with the increase of $h_{2}$, and a maximum $L$ exists (in our case, it is $L=28.9 \mathrm{~mm}$ for $h_{\mathrm{tot}}=h_{1}+h_{2}=4 \mathrm{~mm}$ ).

\section{3. $8 \times 8$ Metasurface Array and Combining Network}

An $8 \times 8$ metasurface array with overall dimensions $163.2 \mathrm{~mm} \times 163.2 \mathrm{~mm} \times 8.867 \mathrm{~mm}$ is depicted in Figure 5(a), 


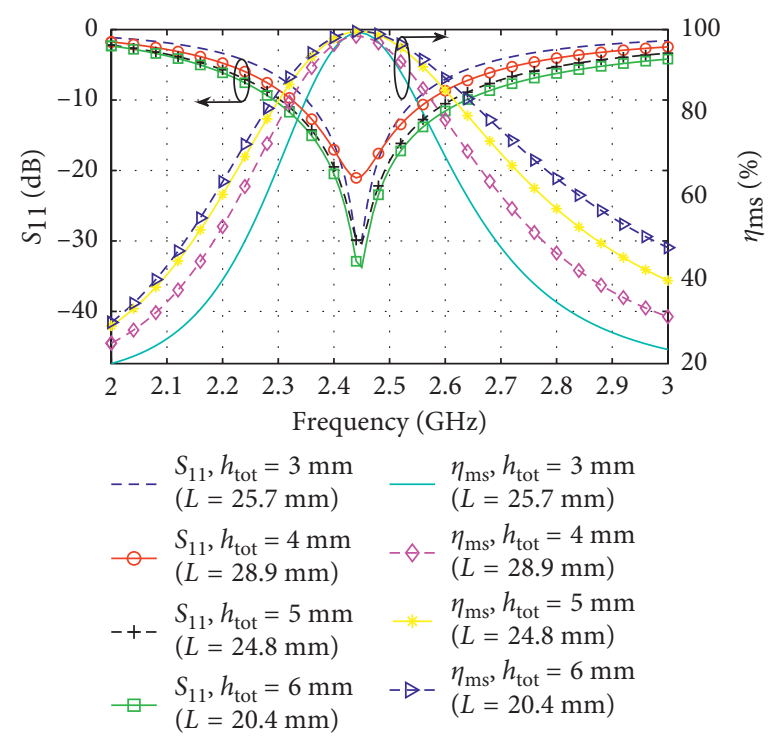

Figure 4: Simulated unit cell $S_{11}$ and efficiency as a function of the air gap $h_{2}$.
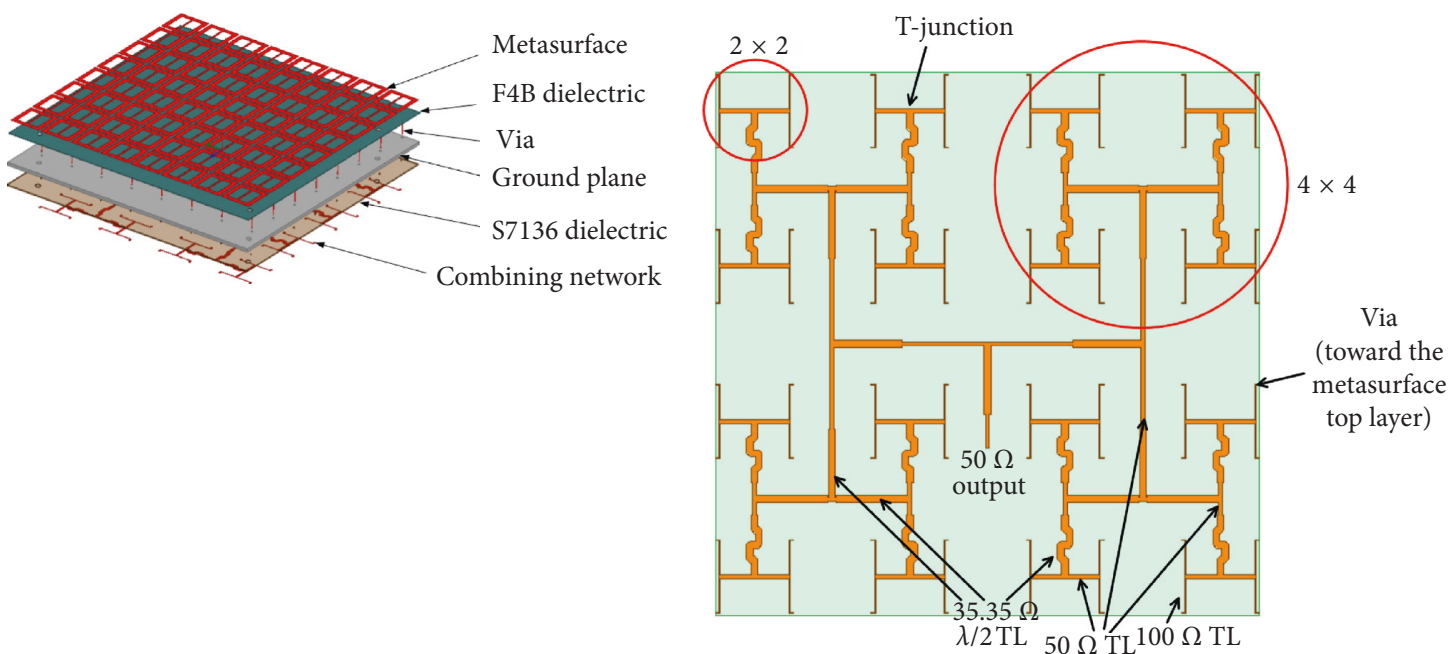

(a)

(b)

FIGURE 5: (a) $8 \times 8$ metasurface array structure and (b) details of the microstrip transmission line- (TL-) based combining network.

designed with the optimized $h_{2}=6 \mathrm{~mm}$ unit cell (air gap thickness has been selected to obtain good impedance matching). The designed structure includes a ground plane of thickness $2 \mathrm{~mm}$ for increasing the overall robustness and a combining network implemented on a $0.508 \mathrm{~mm}$ thick S7136 dielectric $\left(\varepsilon_{\mathrm{R}}=3.6, \tan \delta=0.0035\right)$, shown in Figure 5(b). The optimized dimensions of the unit cell are listed in Table 2.

The combining network is designed with microstrip technology, where matched T-junctions are implemented with quarter-wave transformers [18], and an SMA connector is soldered at the network output (not present in Figure 5(b)). Simulation results of the combining network are shown in Figure 6. For the sake of representation clarity, only maximum, minimum, and average values (selected for each frequencies) of transmission coefficients, i.e.,
Table 2: Optimized dimensions of the $6 \mathrm{~mm}$ air gap unit cells.

\begin{tabular}{lccccccc}
\hline$L$ & $S$ & $w_{1}$ & $w_{2}$ & $g$ & $r$ & $h_{1}$ & $h_{2}$ \\
\hline 20.4 & 1.6 & 1 & 2.45 & 1 & 0.3 & 0.254 & 6 \\
\hline
\end{tabular}

$\max _{i=1, \ldots, 64}\left|S_{i, \text { out }}\right|, \quad \min _{i=1, \ldots, 64}\left|S_{i, \text { out }}\right|, \quad 1 / 64 \times \sum_{i=1}^{64}\left|S_{i, \text { out }}\right|, \quad$ respectively, are shown. Furthermore, phases have been normalized to the first port value, i.e., $\angle S_{i, \text { out }}-\angle S_{1, \text { out }}$, and also in this case only maximum, minimum, and mean values have been depicted.

Assuming an impinging plane wave perpendicular to the collection surface plane, equivalent behaviors among combining network ports (very similar behaviors are found in Figure 6), and neglecting effects caused by the periodicity interruption at the borders (due to the finite size of the 


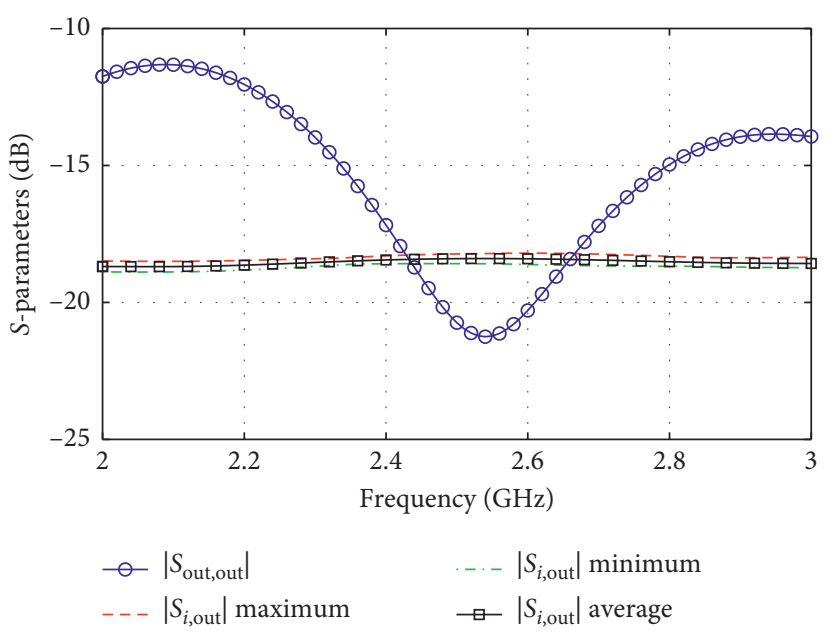

(a)

Figure 6: Simulated $S$-parameters of the $8 \times 8$ combining network.

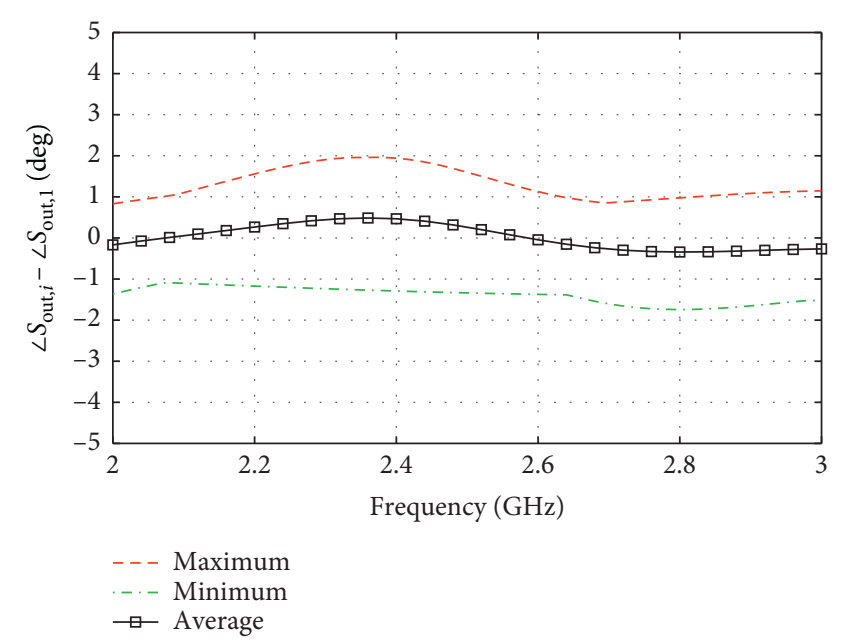

(b) metasurface structure). we estimate an overall efficiency of the structure as $\eta \approx \eta_{\mathrm{ms}} \times \eta_{\mathrm{cn}}=0.876$ at $2.45 \mathrm{GHz}$, where $\eta_{\mathrm{cn}} \approx \mathrm{IL} \cdot\left(1-\left|S_{\text {out,out }}\right|^{2}\right)$ is an estimation of the combining network efficiency which takes into account the network insertion loss IL (the maximum IL is $0.52 \mathrm{~dB}$ at $2.45 \mathrm{GHz}$ and $\left.\eta_{\mathrm{cn}} \approx 0.887\right)$ and the output impedance mismatch. From this simple evaluation, it can be deduced that the combining network insertion loss can severely deteriorate the overall conversion efficiency, and for this reason it has to be minimized during the combining network design. One possibility to reduce the combining network insertion loss (an consequently increase the overall efficiency) is to realize smaller size metasurface arrays, i.e., $2 \times 2$ or $4 \times 4$, and this has been verified by simulation at $2.45 \mathrm{GHz}$ in Figure 7 . Nonetheless, the energy rectification stage usually requires a certain amount of input power to provide the maximum RFto-DC conversion efficiency; for this reason, the energy harvesting system has to be optimally designed to make a trade-off between the metasurface array size increase (for collecting a larger amount of power) and the insertion loss increase (due to the combining network increased size).

Another possibility to reduce the combining network insertion loss is to employ very low-loss dielectric materials to reduce the transmission line signal attenuation. However, vias which connect the combining network and the metasurface can also affect the overall efficiency. Hence, the effect of radius $r$ and length $h_{3}$ of combining network vias to the combining network insertion loss IL and efficiency $\eta_{\mathrm{cn}}$ has been investigated, and simulation results are shown in Figure 8 (it should be noted that the via length $h_{3}$ is assumed to be only the portion of the via within the combining network layer and not the length $h_{\text {tot }}$ within the dual-layer unit cell whose effect is already included in the term $\eta_{\mathrm{ms}}$ ).

While the parameter $r$ has only a minor effect on the combining network IL (at least for the considered range of values), the via length $h_{3}$ increase leads to an IL increase and, consequently, an overall efficiency decrease. This suggests to limit the combining network layer thickness.

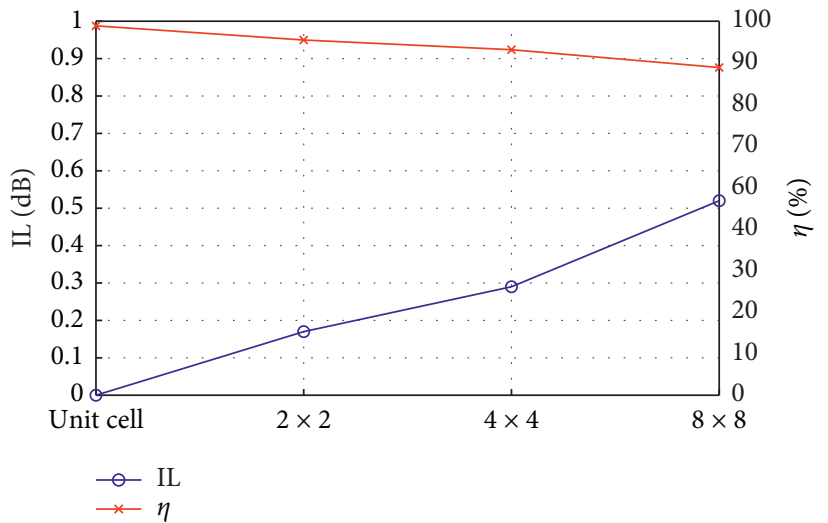

Figure 7: Simulated insertion loss and estimated overall efficiency of the presented array structure at $2.45 \mathrm{GHz}$ as a function of the metasurface array size.

It should be stressed that the above employed overall efficiency formula is an estimation of the efficiency valid only in the case of good impedance matching and perfect phase condition at the input port, which usually happens at the frequency of the efficiency peak. In the next section, we will present the experimental evaluation that includes all possible input mismatches, phase differences among unit cells and combining network, as well as boundary effects caused by the finite size or number of array periodicity.

\section{Measurement Results and Discussion}

A prototype of the $8 \times 8$ metasurface array has been manufactured for experimental verification. Five Teflon spacers fixed with plastic screws are used for improving structure mechanical robustness. Figure 9 shows the experimental setup. It employs an RF signal source capable of providing a power $P_{\mathrm{tx}}=10 \mathrm{dBm}$ within the bandwidth of interest, a horn antenna whose gain and reflection coefficient have been previously characterized in a far-field anechoic chamber, 


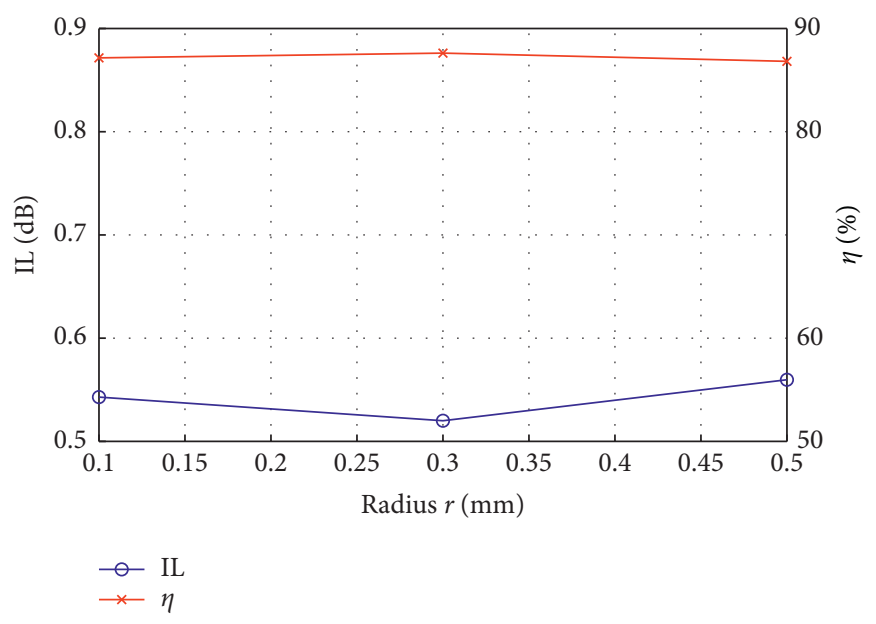

(a)

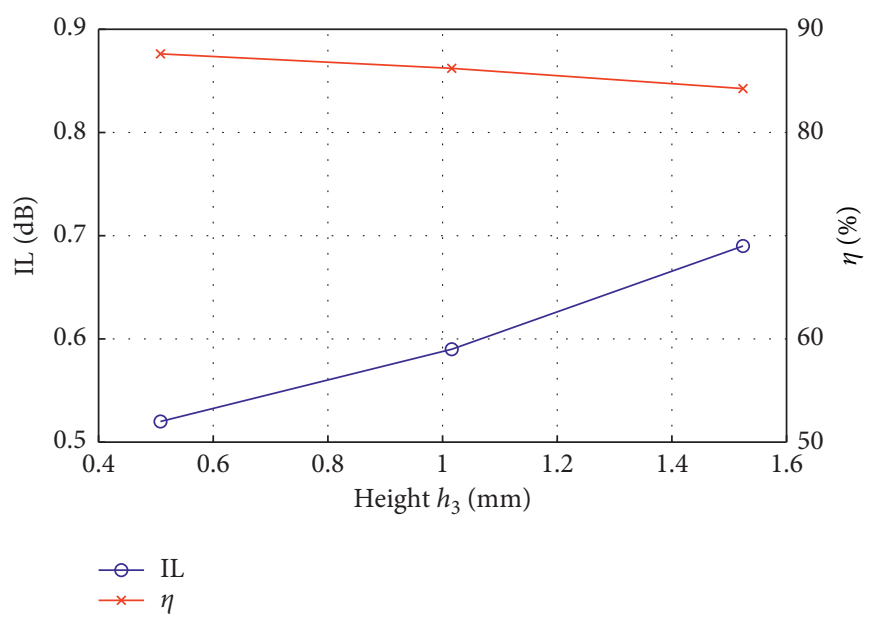

(b)

Figure 8: Simulated insertion loss and estimated overall conversion efficiency of the $8 \times 8$ combining network at $2.45 \mathrm{GHz}$ as a function of (a) via radius $r$ and (b) via length $h_{3}$.

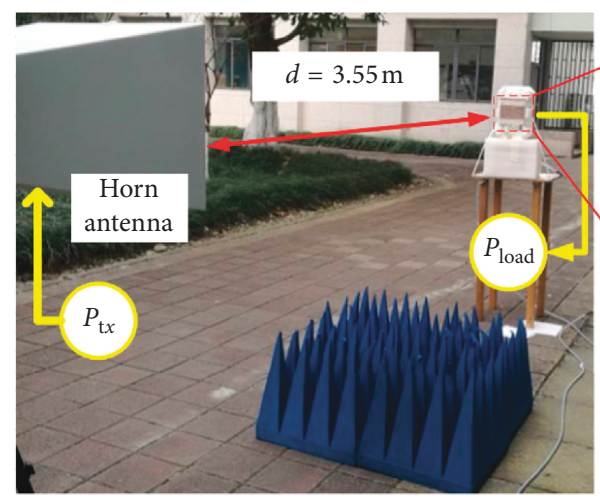

Metasurface

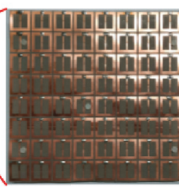

Top view

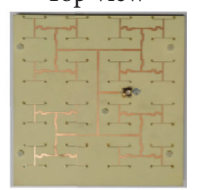

Bottom view

Figure 9: Efficiency measurement setup and the $8 \times 8$ metasurface array prototype.

and a power meter connected to the metasurface output port. The measurement system has been fully calibrated with cable losses and the transmitted power at each frequency point of the measurement.
Measured $S_{\text {out,out }}$ (with a vector network analyzer Agilent N5230A) and absorption efficiency $\eta$ (with the setup depicted in Figure 9) are compared with the simulation results and they are shown in Figure 10. In particular, the metasurface reflection coefficient is about $15.1 \mathrm{~dB}$ at the working frequency $2.45 \mathrm{GHz}$, corroborating well the simulation results.

For the measurement of the efficiency, a distance $d=3.55 \mathrm{~m}$ between the horn antenna and the metasurface is selected which is adequate for the hypothesis of the plane wave. Efficiency is then calculated as

$$
\eta=\frac{P_{\text {load }}}{A_{\text {phys }} \cdot \mathrm{PD}_{\text {inc }}},
$$

where $P_{\text {load }}$ is the power meter reading, $A_{\text {phys }}=26.6 \mathrm{~mm}^{2}$ is the physical area of the metasurface array, and

$$
\mathrm{PD}_{\text {inc }}=\frac{P_{\mathrm{tx}} G_{\mathrm{tx}}}{4 \pi d^{2}},
$$

is the power density impinging at the metasurface. 


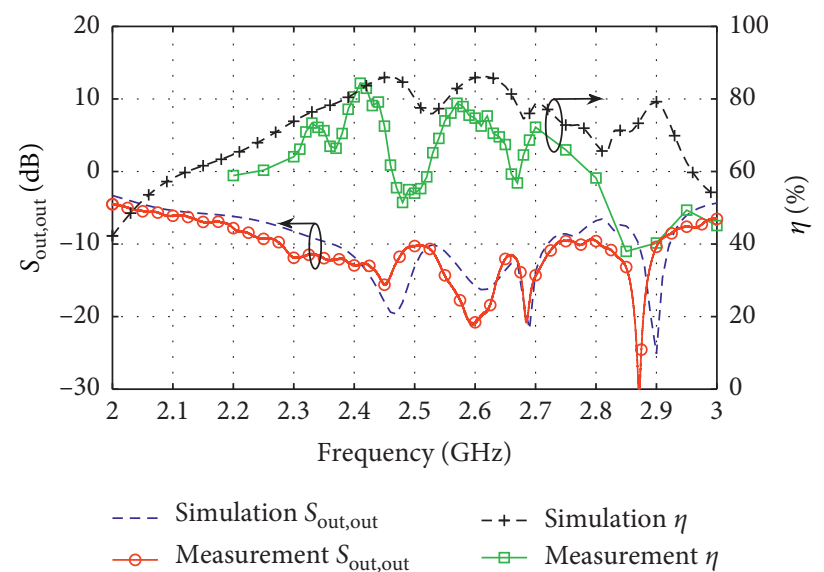

Figure 10: Comparison between simulated and measured $S_{\text {out,out }}$ and efficiency of the $8 \times 8$ metasurface array prototype (efficiency measurement data available from $2.2 \mathrm{GHz}$ ).

TABle 3: Measured performance comparison of the proposed design and other referred metasurfaces.

\begin{tabular}{|c|c|c|c|c|c|}
\hline Ref. & Structure & Efficiency (\%) & $\begin{array}{l}\text { Material (metasurface, } \\
\text { combining network) }\end{array}$ & $\begin{array}{c}\text { Unit-cell area }\left(\mathrm{mm}^{3}\right) \text {, thickness } \\
{[\lambda]}\end{array}$ & $\begin{array}{c}\text { Cost } \\
\text { (unit cell) }\end{array}$ \\
\hline [7] & G-CSRR $(11 \times 11)$ & $83 \%(5.55 \mathrm{GHz})^{*}$ & Rogers duriod RT5880, - & $342.25,0.015^{\dagger}$ & High \\
\hline [8] & ELC $(13 \times 13)$ & $93 \%(3 \mathrm{GHz})^{*}$ & Rogers TMM10i, - & $56.25,0.026^{\dagger}$ & High \\
\hline$[16]$ & $\begin{array}{c}\text { SRR }+ \text { hollow cylinder } \\
\qquad(10 \times 10)\end{array}$ & $\begin{array}{c}97.3 \% \\
(2.45 \mathrm{GHz})^{*}\end{array}$ & Copper + PTFE, - & $400,0.074^{\dagger}$ & High \\
\hline [17] & Split-loop on air $(20 \times 20)$ & $97 \%(2.45 \mathrm{GHz})^{*}$ & Air, - & $392,0.067^{\dagger}$ & Low \\
\hline$[18]$ & ERR $(8 \times 8)$ & $78 \%(3 \mathrm{GHz})$ & Rogers TMM10i, Rogers 5880 & $232.56,0.016^{\dagger}$ & High \\
\hline [19] & $\operatorname{ELC}(8 \times 8)$ & $86 \%(2.45 \mathrm{GHz})$ & $\begin{array}{c}\text { Rogers RO4350, Rogers } \\
\text { RO4350 }\end{array}$ & $240.25,0.051$ & High \\
\hline Prop. & $\operatorname{ELC}(8 \times 8)$ & $84.4 \%(2.41 \mathrm{GHz})$ & F4B/air, S7136 & $484,0.071$ & Low \\
\hline
\end{tabular}

Figure 10 shows some differences from Figure 3 because simulation in Figure 10 takes into account the whole structure (metasurface + combining network) and for this reason includes the effect of the combining network insertion loss IL described in Section 3 and other combining network design imperfections shown in Figure 6, and the metasurface periodicity interruption due to the finite size (which can be roughly quantified by comparing the efficiency peak estimated in Section 3-87.6\%-and the simulated efficiency peak in Figure 10-85.9\%). Moreover, Figure 10 presents a measured peak efficiency of $84.4 \%$ at $2.41 \mathrm{GHz}$. Discrepancies between the simulation and measurement results are mainly due to fabrication imperfections (since the dielectric material is very thin, it is not perfectly parallel with the ground plane but it has a slightly undulating profile), variations of material parameters from their nominal values (for both the metasurface and the combining network layers), and measurement setup imperfections (such as nonperfect antenna and metasurface alignment and transmitting antenna gain $G_{\mathrm{tx}}$ calibration errors).

Finally, the measured performance of the proposed metasurface array is compared with that of other recently proposed structures ( ${ }^{*}$ indicates absorption efficiency measurement performed only on a unit cell; ${ }^{\dagger}$ indicates that thickness is given only for the unit cell without combining network) and the results are shown in Table 3 . It should be noted that the most part of the available literature only provides results in terms of unit-cell efficiency, without considering harvester practical implementation aspects such as combining network insertion loss and array periodicity interruption. Nonetheless, from Table 3 it can be deduced that the employment of an air gap structure for our design is beneficial to costs and overall efficiency (larger than that of [18] and very similar to that of [19]), even if, in general, the use of a suspended structure might increases thickness and fabrication complexity (since vias have to be connected through air). Nevertheless, the proposed metasurface enhances the wireless power collection with respect to the other referred structures because of a larger collection surface and, consequently, achieves improved overall energy harvesting performance. In fact, assuming to employ two structures as in [19] to obtain a collection surface close to the one proposed, i.e., to collect the same amount of power which can be collected by employing the proposed metasurface, and to design a new combining network to combine the two structures collected power into one rectifier device, the total efficiency will decrease due to the new combining network insertion loss, which has been demonstrated in Section 3 to be the most detrimental effect.

\section{Conclusion}

An air gap structure useful for the design of a metasurface unit cell for energy harvesting applications has been 
proposed. Advantages in terms of costs and absorption efficiency have been demonstrated by employing a classical electric $L C$ unit cell, even if the proposed air gap structure can be easily extended to many other unit cells. Furthermore, an $8 \times 8$ metasurface array has been designed and prototyped, showing an efficiency of $84.4 \%$, and with a larger surface which can be exploited to collect a larger amount of power and, consequently, to improve the overall harvesting efficiency.

\section{Data Availability}

The simulation and measurement data used to support the findings of this study are available from the corresponding author upon request.

\section{Conflicts of Interest}

Authors declare that there are no conflicts of interest regarding the publication of this paper.

\section{Acknowledgments}

This work was supported in part by the National Natural Science Foundation of China under project contracts nos. 61601093, 61701082, 61701116, 61971113, and 61901095, in part by National Key R\&D Program under project contract SQ2018YFB180088, in part by Guangdong Provincial Research and Development Plan in Key Areas under project contract nos. 2019B010141001 and 2019B010142001, in part by Sichuan Provincial Science and Technology Planning Program of China under project contracts nos. 2018HH0034, 2019YFG0418, and 2019YFG0120, and in part by Science and Technology on Electronic Information Control Laboratory.

\section{References}

[1] P. E. Glaser, "Power from the sun: its future," Science, vol. 162, no. 3856, pp. 857-861, 1968.

[2] A. Massa, G. Oliveri, F. Viani, and P. Rocca, "Array designs for long-distance wireless power transmission: state-of-the-art and innovative solutions," Proceedings of the IEEE, vol. 101, no. 6, pp. 1464-1481, 2013.

[3] B. Strassner and K. Chang, "Microwave power transmission: historical milestones and system components," Proceedings of the IEEE, vol. 101, no. 6, pp. 1379-1396, 2013.

[4] R. Cicchetti, A. Faraone, and O. Testa, "Energy-based representation of multiport circuits and antennas suitable for near- and far-field syntheses," IEEE Transactions on Antennas and Propagation, vol. 67, no. 1, pp. 85-98, 2019.

[5] A. Buffi, P. Nepa, and G. Manara, "Design criteria for nearfield-focused planar arrays," IEEE Antennas and Propagation Magazine, vol. 54, no. 1, pp. 40-50, 2012.

[6] C. R. Valenta and G. D. Durgin, "Harvesting wireless power: survey of energy-harvester conversion efficiency in far-field, wireless power transfer systems," IEEE Microwave Magazine, vol. 15, no. 4, pp. 108-120, 2014.

[7] B. Alavikia, T. S. Almoneef, and O. M. Ramahi, "Complementary split ring resonator arrays for electromagnetic energy harvesting," Applied Physics Letters, vol. 107, no. 3, Article ID 033902, 2015.
[8] T. S. Almoneef and O. M. Ramahi, "Metamaterial electromagnetic energy harvester with near unity efficiency," Applied Physics Letters, vol. 106, no. 15, Article ID 153902, 2015.

[9] X. Duan, X. Chen, and L. Zhou, "A metamaterial electromagnetic energy rectifying surface with high harvesting efficiency," AIP Advances, vol. 6, no. 12, Article ID 125020, 2016.

[10] X. Zhang, H. Liu, and L. Li, "Tri-band miniaturized wideangle and polarization-insensitive metasurface for ambient energy harvesting," Applied Physics Letters, vol. 111, no. 7, Article ID 071902, 2017.

[11] S. Shang, S. Yang, M. Shan, J. Liu, and H. Cao, "High performance metamaterial device with enhanced electromagnetic energy harvesting efficiency," AIP Advances, vol. 7, no. 10, Article ID 105204, 2017.

[12] H. T. Zhong, X. X. Yang, C. Tan, and K. Yu, “Triple-band polarization-insensitive and wide-angle metamaterial array for electromagnetic energy harvesting," Applied Physics Letters, vol. 109, no. 25, Article ID 253904, 2016.

[13] H. T. Zhong, X. X. Yang, X. T. Song, Z. Y. Guo, and F. Yu, "Wideband metamaterial array with polarization-independent and wide incident angle for harvesting ambient electromagnetic energy and wireless power transfer," Applied Physics Letters, vol. 111, no. 21, Article ID 213902, 2017.

[14] F. Yu, X. Yang, H. Zhong, C. Chu, and S. Gao, "Polarizationinsensitive wide-angle-reception metasurface with simplified structure for harvesting electromagnetic energy," Applied Physics Letters, vol. 113, no. 12, Article ID 123903, 2018.

[15] X. Zhang, H. Liu, and L. Li, "Electromagnetic power harvester using wide-angle and polarization-insensitive metasurfaces," Applied Sciences, vol. 8, no. 4, 2018.

[16] X. Duan, X. Chen, Y. Zhou, L. Zhou, S. Hao, and S. Hao, "Wideband metamaterial electromagnetic energy harvester with high capture efficiency and wide incident angle," IEEE Antennas and Wireless Propagation Letters, vol. 17, no. 9, pp. 1617-1621, 2018.

[17] S. Y. Wang, P. Xu, W. Geyi, and Z. Ma, "Split-loop resonator array for microwave energy harvesting," Applied Physics Letters, vol. 109, no. 20, Article ID 203903, 2016.

[18] M. El Badawe, T. S. Almoneef, and O. M. Ramahi, "A metasurface for conversion of electromagnetic radiation to DC," AIP Advances, vol. 7, no. 3, Article ID 035112, 2017.

[19] P. Xu, S.-Y. Wang, and W. Geyi, "Design of an effective energy receiving adapter for microwave wireless power transmission application," AIP Advances, vol. 6, no. 10, Article ID 105010, 2016.

[20] T. S. Almoneef, F. Erkmen, M. A. Alotaibi, and O. M. Ramahi, "A new approach to microwave rectennas using tightly coupled antennas," IEEE Transactions on Antennas and Propagation, vol. 66, no. 4, pp. 1714-1724, 2018.

[21] W. Hu, D. Inserra, Y. Huang et al., "High efficiency electromagnetic energy harvesting with metasurface," in Proceedings of the IEEE Asia-Pacific Conference on Antennas and Propagation (APCAP), Auckland, New Zealand, August 2018.

[22] D. Schurig, J. J. Mock, and D. R. Smith, "Electric-field-coupled resonators for negative permittivity metamaterials," Applied Physics Letters, vol. 88, no. 4, Article ID 041109, 2006. 


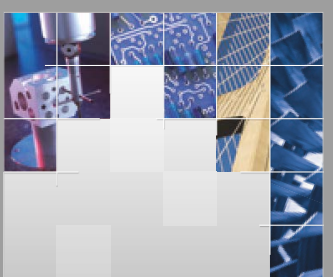

\section{Enfincering}
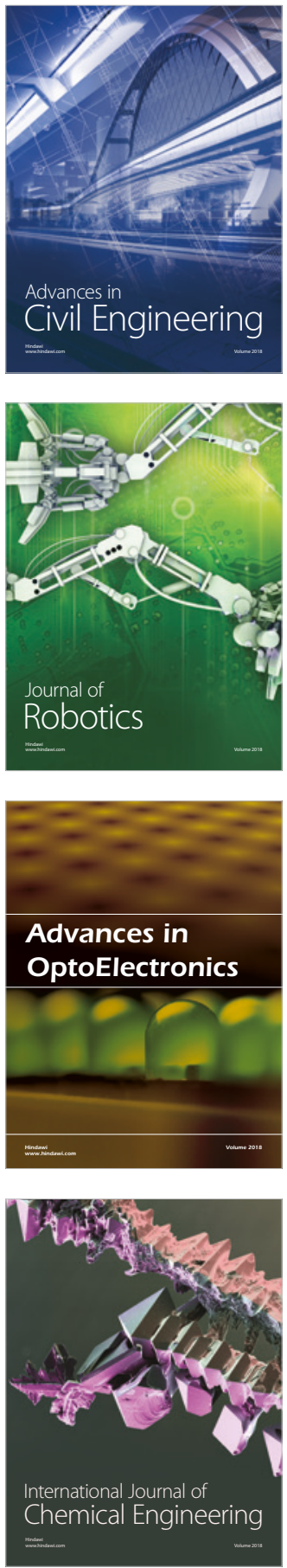

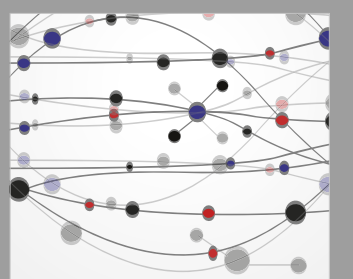

\section{Rotating \\ Machinery}

The Scientific World Journal

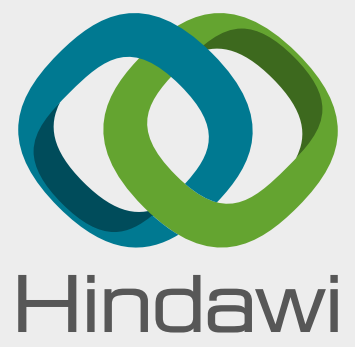

Submit your manuscripts at

www.hindawi.com
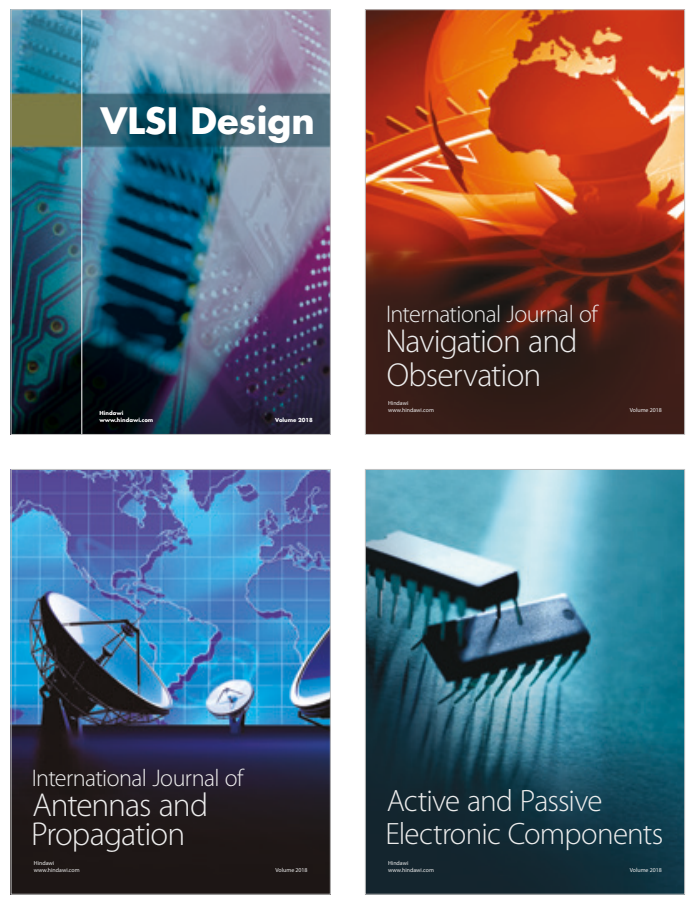
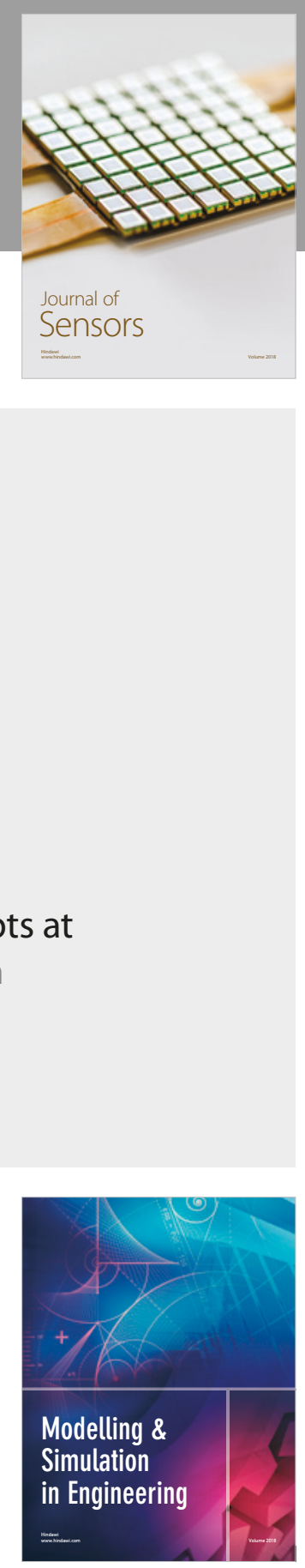

\section{Advances \\ Multimedia}
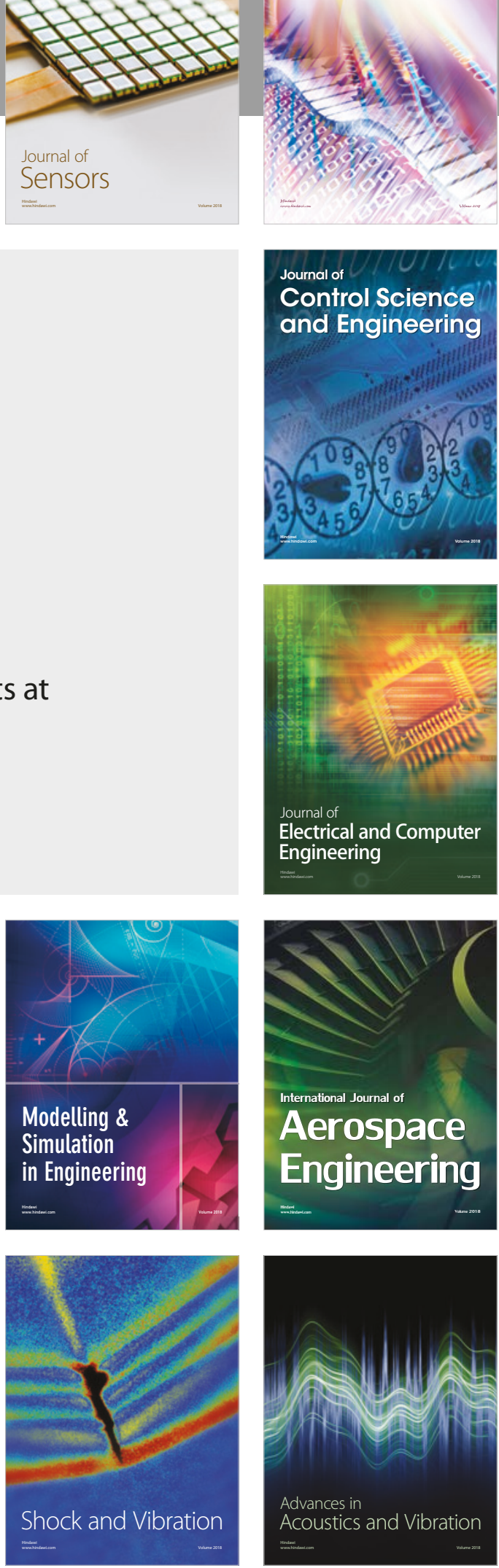